\title{
Influence de l'air occlus et dissous dans l'eau sur le comportement des pompes en cavitation
}

\author{
B. Le Fur (Mme) J. F. David D. Pécot
}

CETIM, Nantes

\section{Origine de la recherche}

Suite à une demande des constructeurs de pompes, une recherche bibliographique axée sur le thème: Influence des paramètres suivants : échelle géométrique, vitesse de rotation, teneur en air, effet thermodynamique, sur le comportement des pompes en cavitation, a été effectuée par le CETIM de Nantes en 1983-84. Cette recherche bibliographique avait pour but d'orienter une recherche expérimentale.

Une fois analysée et synthétisée toute la bibliographie obtenue, il a été décidé, en accord avec la profession, de conduire une recherche expérimentale sur l'influence de la teneur en air occlus et dissous sur la cavitation des pompes.

\section{Programme expérimental}

Le programme expérimental comprend des essais sur 2 pompes de $N S$ respectifs 20 et 80 .

Ces pompes sont montées tantôt en boucle ouverte pour étude de l'influence de l'air occlus sur la cavitation, tantôt en boucle fermée pour étude de l'influence de l'air dissous sur la cavitation.

Les résultats expérimentaux présentés ici concernent les essais relatifs à l'influence de l'air occlus sur le comportement en cavitation de la pompe de NS 80 et ceux relatifs à l'influence de l'air dissous sur le comportement en cavitation de la pompe NS 20. Les deux autres phases d'essais (pompe NS 20 en boucle ouverte et pompe NS 80 en boucle fermée) sont actuellement en cours.
3. Pompe NS 80 en boucle ouverte (air occlus)

\subsection{La boucle}

La pompe a des diamètres d'aspiration et de refoulement égaux et de $D N 300$. Elle aspire dans un puits de $10 \mathrm{~m}$ de profondeur et refoule dans le bassin.

L'injection d'air se fait à $300 \mathrm{~mm}$ de la bride d'aspiration à travers une grille percée de quantité de petits trous de $2 / 10^{\mathrm{c}}$.

La manchette de $300 \mathrm{~mm}$ de long située entre la bride d'aspiration et la grille est en plexiglass de même que celle de $600 \mathrm{~mm}$ de long et toujours de $D N 300$ située en amont de la grille.

L'aspiration de la pompe est telle que l'on a une très bonne visualisation de la roue.

La ligne d'aspiration est équipée d'une vanne de réglage située très en aval de la pompe et de tuyauteries de $D N 300-400-500$ et 600 .

La ligne de refoulement comprend des tuyauteries de $D N 300$ et 400 et un débitmètre à hélice de $2000 \mathrm{~m}^{3} / \mathrm{h}$.

La pompe est entraînée par un moteur courant continu de $460 \mathrm{~kW}$.

\subsection{Les essais}

Les essais de cavitation sont faits par baisse du plan d'eau pour des raisons de qualité d'eau.

Les essais sont effectués à 3 vitesses de rotation :

$$
\begin{gathered}
723 \mathrm{tr} / \mathrm{min} \\
\left(Q_{N}=820 \mathrm{~m}^{3} / \mathrm{h}\right)\left(Q_{N}=975 \mathrm{~m}^{3} / \mathrm{h}\right)\left(Q_{N}=1240 \mathrm{~m}^{3} / \mathrm{h}\right)
\end{gathered}
$$

\section{Effect of occluded air dissolved in water regarding the behaviour of cavitation pumps.}

This article presents test results showing : firstly the influence of the presence of occluded air (within a volume percentage varying from 0 to $5 \%$ on the cavitation behaviour of a NS 80 pump and this includes $3 \mathrm{rpm}$ and 7 flow rate points per rpm. Secondly, the effect of the percentage of air dissolved in water concerning the cavitation behaviour of a NS 20 pump and this includes $3 \mathrm{rpm}$ and 5 flow rate points per rpm. 
1.
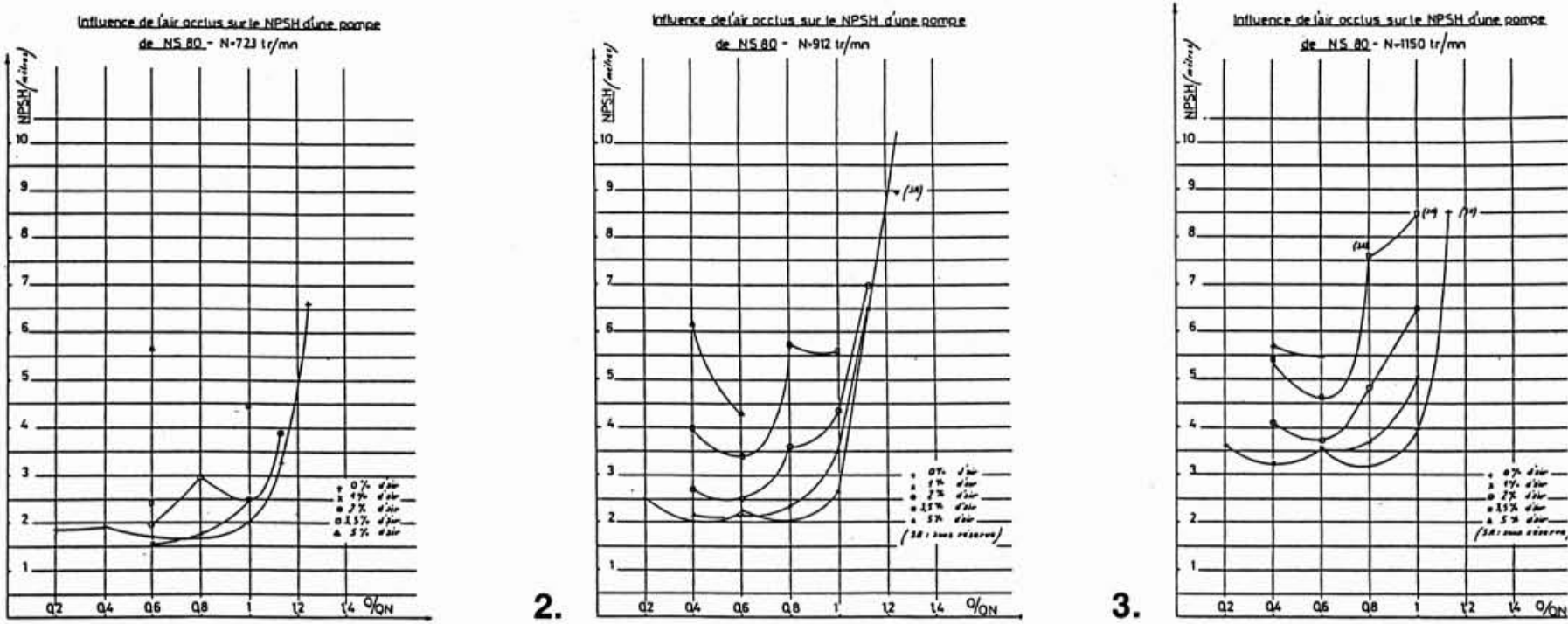

2.
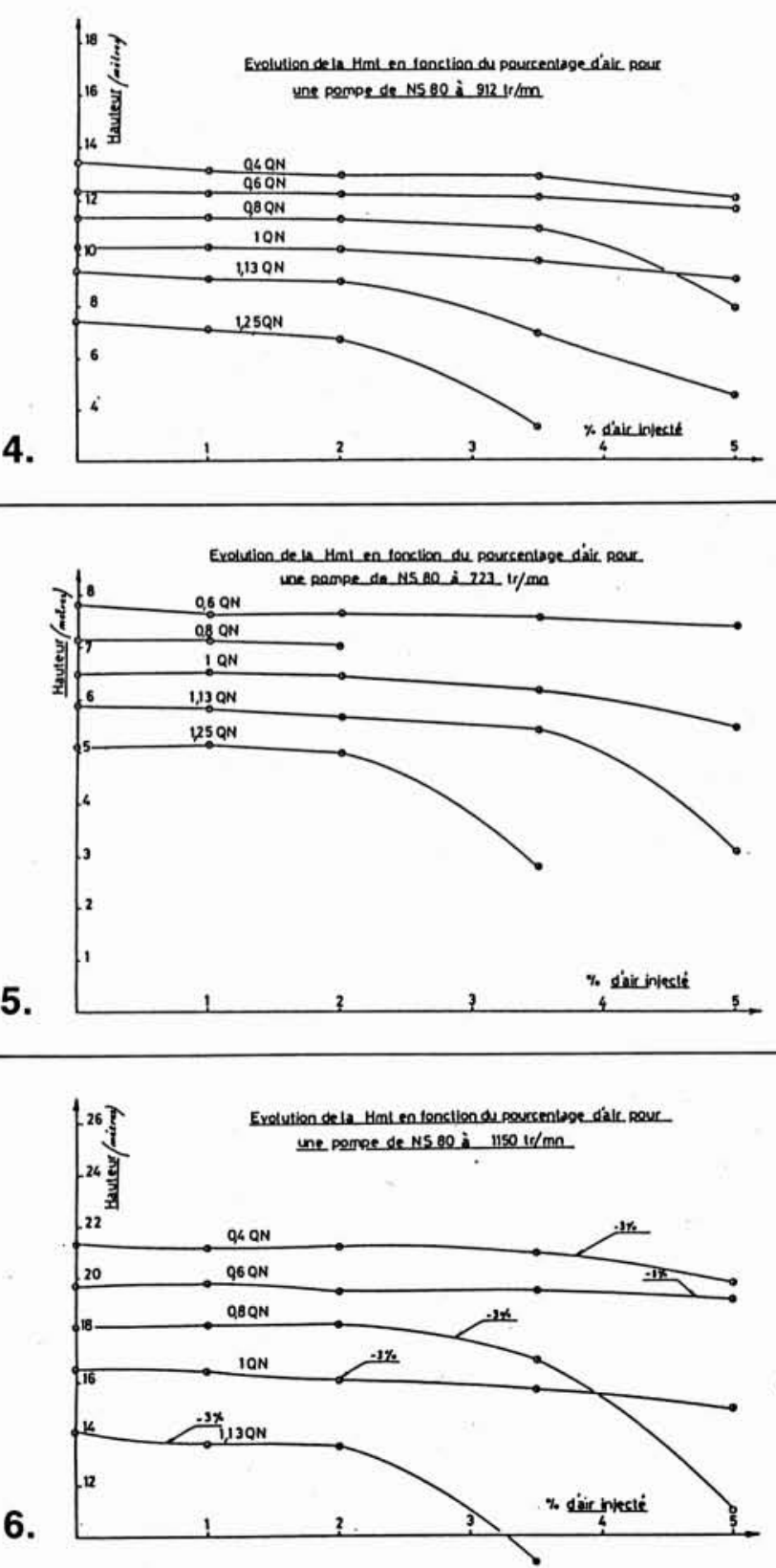

avec à chaque vitesse 7 points de débit.

$$
Q / Q_{N}=0,2-0,4-0,6-0,8-1-1,13-1,25
$$

(le point de débit nominal correspond au point de rendement maximum de la pompe) et à chaque point 5 valeurs du pourcentage volumique d'air ramené à la pression d'aspiration de la pompe. $\alpha=0 \%-1 \%-2 \%-3,5 \%-5 \%$.

\subsection{Les mesures}

La majeure partie des mesures est faite par acquisition de données sinon les valeurs mesurées sont rentrées manuellement au clavier. Ces mesures sont traitées aussitôt par le calculateur qui fournit :

- les caractéristiques classiques de la pompe (vitesse de rotation, couple, débit, hauteur, puissance, rendement, pression amont, NPSH disponible);

- les grandeurs nécessaires au calcul du taux volumique d'air injecté ;

- le calcul du taux volumique d'air injecté ramené à la pression d'aspiration de la pompe et si besoin des indications de réglage.

\subsection{Mesure et calcul du taux volumique d'air injecté}

Le taux volumique d'air injecté ramené à la pression d'aspiration de la pompe est égal au rapport du débit d'air injecté ramené à la pression d'aspiration de la pompe au débit d'eau. Le débit d'air injecté est mesuré à l'aide d'un tube de Pitot placé sur la ligne d'injection d'air qui aboutit sur la grille.

La lecture du niveau incliné permet de connaître ce débit d'air injecté, à la pression d'injection d'air et à la température de l'air injecté.

La température de l'eau et la pression à l'aspiration de la pompe étant connues il est facile d'en déduire le débit 
d'air injecté ramené à la pression d'aspiration de la pompe et à la température de l'eau. Le réglage de ce débit d'air pour obtention des pourcentages $1 \%-2 \%$ $3,5 \%-5 \%$ se fait à partir de la pression d'injection d'air. Ce réglage suppose un étalonnage préalable du Pitot.

\subsection{Résultats}

Les résultats sont présentés par vitesse de rotation sous forme d'évolution du NPSH $3 \%$ en fonction du rapport $Q / Q_{N}$ pour l'ensemble des 5 pourcentages d'air ( $f$ ig. 1 : $N=723$, fig. $2: N=912$, fig. $3: N=1150$ ).

Il manque quelques points pour les grands débits avec en général les gros pourcentages d'air. Les points n'ont pu être obtenus parce que les NPSH étaient trop hauts (les essais étant faits en boucle ouverte en aspiration sur le plan d'eau, nous sommes limités en disponible).

Les points à faibles débits $\left(0,2 Q_{N}\right.$ aux 3 vitesses et $0,4 Q_{N}$ à $723 \mathrm{tr} / \mathrm{min}$ ) n'ont pu être faits à cause de la prérotation trop importante qui empêchait tout réglage de l'injection d'air.

La hauteur prise comme référence pour le calcul du NPSH pour un pourcentage d'air donné est la hauteur manométrique totale obtenue avec ce même pourcentage d'air. L'évolution de la hauteur manométrique totale (pour le niveau le plus haut du bassin) avec le pourcentage d'air injecté est montrée à titre d'exemple sur les figures 4,5 et 6 .

Au vu de ces résultats, il apparaît que le comportement de la pompe $N S 80$ hors cavitation (en particulier la $\mathrm{Hmt}$ ) est tout d'abord altéré vers les grands débits pour des pourcentages d'air de l'ordre de 2-2,5\%. Ensuite c'est la tranche autour de $0,8 Q_{N}$ qui est touchée mais pour des pourcentages d'air supérieurs (de l'ordre de $3,5 \%-4 \%$ ). Vers $5 \%$, l'ensemble des points de débit voient leur $\mathrm{Hmt}$ diminuer.

Par contre le comportement de la pompe NS 80 en cavitation semble être modifié pour des pourcentages moindres. Un pourcentage d'air compris entre 1 et $1,5 \%$ peut altérer le NPSH autour de la tranche $0,8 Q_{N}-1 Q_{N}$. Il faut atteindre des pourcentages d'air de l'ordre de $2-2,5 \%$ pour altérer le $N P S H$ dans les faibles débits, soit dans la tranche autour de $0,4 Q_{N}-0,6 Q_{N}$.

\section{Pompe NS 20 en boucle fermée (air dissous)}

\subsection{La boucle}

La pompe a un diamètre de roue égal à $330 \mathrm{~mm}$ et possède 10 aubes. L'aspiration de la pompe est en $D N 125$ et le refoulement en $D N 100$. La boucle est une boucle fermée composée d'éléments de tuyauteries de $D N$ 100-125-150 dont une manchette $D N 125$ en plexiglas à l'aspiration de la pompe et d'une bonbonne de tranquillisation et dépressurisation. Elle comprend aussi outre une vanne et un débitmètre électromagnétique de $450 \mathrm{~m}^{3} / \mathrm{h}$ un module de désaération de l'eau monté en parallèle. Ce module consiste en un hydroéjecteur qui prend l'eau de la boucle pour la réinjecter en pluie audessus du niveau d'eau de la bonbonne.
La mesure du taux d'oxygène dissous qui sert à quantifier la quantité d'air dissous dans l'eau, se fait en continu à l'aide d'un oxymètre. Cet oxymètre est monté en dérivation entre les lignes d'aspiration et de refoulement. Cette mesure du taux d'oxygène dissous à partir de l'oxymètre est régulièrement contrôlée par mesure chimique.

\subsection{Les essais}

Les essais de cavitation sont faits par dépressurisation de la boucle. Ils sont effectués à 3 vitesses de rotation :

$$
\begin{gathered}
875 \mathrm{tr} / \min \left(Q_{N}=85 \mathrm{~m}^{3} / \mathrm{h}\right), \\
975 \mathrm{tr} / \min \left(Q_{N}=95 \mathrm{~m}^{3} / \mathrm{h}\right), \\
1470 \mathrm{tr} / \min \left(Q_{N}=143 \mathrm{~m}^{3} / \mathrm{h}\right),
\end{gathered}
$$

avec 5 points de débit par vitesse correspondants aux rapports :

$$
Q / Q_{N}=0,6-0,8-1-1,25-1,5 .
$$

A chaque point, les essais sont faits pour 3 valeurs du taux initial d'oxygène dissous (taux avant dépressurisation de la boucle pour essai de cavitation). Ces 3 valeurs sont choisies de telle sorte à avoir :

- un taux initial proche de la saturation ;

- un taux initial correspondant à une eau bien désaérée ;

- un taux initial intermédiaire entre les 2 valeurs précédentes.

Le taux d'oxygène dissous diminue au fur et à mesure que l'on fait baisser la pression. Sont donc notés à chaque essai de cavitation le taux initial d'oxygène dissous, le taux autour du point correspondant au NPSH à $3 \%$, le taux en fin d'essai.

\subsection{Résultats}

Les résultats des essais sont synthétisés dans le tableau cidessous.

Au vu de ces résultats, il semble qu'il n'y ait pas de

\begin{tabular}{|c|c|c|c|c|c|c|c|c|c|c|c|c|c|c|c|}
\hline \multicolumn{16}{|l|}{ Vitesse 875 tr/mn } \\
\hline Q/ON & \multicolumn{3}{|c|}{1.55} & \multicolumn{3}{|c|}{1,325} & \multicolumn{3}{|c|}{1} & \multicolumn{3}{|c|}{0,835} & & & \\
\hline $\begin{array}{l}\text { Taux } 02 \text { initial } \\
\text { Taux } 02 \text { d } 3 \% \\
\text { Taux } 02 \text { final }\end{array}$ & $\begin{array}{l}10,3 \\
9,7 \\
8,7\end{array}$ & $\begin{array}{l}6,7 \\
6,7 \\
6.6\end{array}$ & $\begin{array}{l}4,6 \\
4,6 \\
4,6\end{array}$ & $\begin{array}{l}10,4 \\
7,8 \\
6,5\end{array}$ & $\begin{array}{l}7.2 \\
6.4 \\
6.2\end{array}$ & $\begin{array}{l}3,5 \\
3,5 \\
3,5\end{array}$ & $\begin{array}{l}10,3 \\
=5 \\
4,2\end{array}$ & $\begin{array}{l}8,6 \\
=4 \\
3,6\end{array}$ & $\begin{array}{c}4,6 \\
=3,5 \\
2,6\end{array}$ & $\begin{array}{c}10,3 \\
4 \\
2,8\end{array}$ & $\begin{array}{r}6,9 \\
3 \\
2,3\end{array}$ & $\begin{array}{l}3,8 \\
2,2 \\
1,8\end{array}$ & & & \\
\hline $\mathrm{NPSH} 35(m)$ & 7,50 & 7,53 & 7,53 & 4,12 & 4.05 & 4,12 & 1,53 & 1.51 & 1,43 & 1.16 & 1.18 & 1.17 & & & \\
\hline \multicolumn{16}{|l|}{ Vitesse: 975 uimn } \\
\hline Q/ON & \multicolumn{3}{|c|}{1.55} & \multicolumn{3}{|c|}{1,325} & \multicolumn{3}{|c|}{1.04} & \multicolumn{3}{|c|}{0,835} & \multicolumn{3}{|c|}{0.615} \\
\hline $\begin{array}{l}\text { Toux } 022 \text { intital } \\
\text { Taux } 22.43 \\
\text { Taux } 02 \text { thinal }\end{array}$ & $\begin{array}{l}10 \\
9,6 \\
8,6\end{array}$ & $\begin{array}{l}5 \\
4.9 \\
4,7\end{array}$ & $\begin{array}{l}3.2 \\
3,1\end{array}$ & $\begin{array}{l}10 \\
7.7 \\
5,2\end{array}$ & $\begin{array}{l}7.2 \\
7.9 \\
4.9\end{array}$ & $\begin{array}{l}4,6 \\
4,3\end{array}$ & $\begin{array}{l}10,3 \\
5,7 \\
4,4\end{array}$ & $\begin{array}{l}7 \\
4,4 \\
3,2\end{array}$ & $\begin{array}{l}4,1 \\
3,2 \\
2,05\end{array}$ & $\begin{array}{l}10.2 \\
2,8 \\
2,4\end{array}$ & $\begin{array}{l}6,2 \\
2,3 \\
1,8\end{array}$ & $\begin{array}{l}3 \\
1.6 \\
1.4\end{array}$ & $\begin{array}{l}10,2 \\
1,2 \\
0,8\end{array}$ & $\begin{array}{l}5.5 \\
1.2\end{array}$ & $\begin{array}{l}3,2 \\
1.3\end{array}$ \\
\hline $\mathrm{NPSH} 3 \%(\mathrm{~m})$ & 9,95 & 9,95 & 9,88 & 5,81 & 5,82 & 5,80 & 1,99 & 2,09 & 2,05 & 1,13 & 1,14 & 1,17 & 0,87 & 0.90 & 0,90 \\
\hline \multicolumn{16}{|c|}{ Vitesse: $1475 \mathrm{t} / \mathrm{mn}$} \\
\hline QMON & & & & & 1,32 & & & 1,04 & & & 0,835 & & & 0,615 & \\
\hline $\begin{array}{l}\text { Taux } 02 \text { initial } \\
\text { Taux } 0223^{3} 3 \\
\text { Taux } 02 \text { tinal }\end{array}$ & & & & $\begin{array}{l}8 \\
8 \\
8\end{array}$ & $\begin{array}{l}6.8 \\
6.8 \\
6.7\end{array}$ & & $\begin{array}{l}8.6 \\
6,3 \\
4,7\end{array}$ & $\begin{array}{l}8.1 \\
5,6 \\
4,2\end{array}$ & $\begin{array}{l}5,7 \\
5,2 \\
4,4\end{array}$ & $\begin{array}{l}9,2 \\
2,4 \\
1,4\end{array}$ & $\begin{array}{l}8.7 \\
3.5\end{array}$ & $\begin{array}{l}4,7 \\
2,5 \\
1,3\end{array}$ & $\begin{array}{l}10.9 \\
4,2 \\
3,3\end{array}$ & $\begin{array}{l}7.8 \\
3,6 \\
2,4\end{array}$ & $\begin{array}{l}4,7 \\
2,6 \\
2\end{array}$ \\
\hline $\mathrm{NPSH} 3 \%(\mathrm{~m})$ & & & & 12,85 & 12,70 & & 4,66 & 4,45 & 4,46 & 2,22 & 2,18 & 2,23 & 1,96 & 2,05 & 1.90 \\
\hline
\end{tabular}
variation notable du NPSH industriel avec le taux d'oxygène dissous pour la pompe NS 20 .

INFLUENCE DE LA TENEUR EN OXYGENE DISSOUS SUR LE NPSH 3 \% D'UNE POMPE DE NS 20 


\section{Conclusion}

Ces essais permettent de quantifier l'influence de la présence d'air occlus sur le NPSH d'une pompe de $N S 80$ et de voir que le taux d'oxygène dissous dans l'eau n'a pas d'influence notable sur le NPSH d'une pompe de NS 20.

Les essais actuellement en cours permettront de voir l'influence de la présence d'air occlus sur le $N P S H$ de la pompe $N S 20$ et l'influence du taux d'air dissous sur le $N P S H$ de la pompe NS 80 .

\section{Notations}

$\begin{array}{ll}D N & : \text { Diamètre nominal. } \\ N & : \text { Vitesse de rotation (tr/min). } \\ Q_{N} & : \text { Débit nominal. } \\ H \text { ou Hmt } & : \text { Hauteur manométrique totale (mètres). } \\ N P S H & : \text { Net positive suction head (mètres). } \\ N S & : \text { Vitesse spécifique }\end{array}$

$$
N S=\underset{(\operatorname{tr} / \mathrm{min})}{N} \underset{\left(\mathrm{m}^{3} / \mathrm{s}\right)}{\stackrel{Q}{N} / H_{(\mathrm{m})}^{3 / 4}} .
$$

\section{Références}

F. G. HAMITT, Effects of gas content upon cavitation inception, performance and damage, Journal of Hydraulic Research, vol. 10, n 3, 1972, pp. 259-290.

I. J. KaRASSIK, Centrifugal pump clinic $1 / 83$, World pumps, nr 2, Février 1983.

Murakami Minemura, Effects of entrained air on the performance of Centrifugal pumps under cavitating conditions, Bulletin of the JSME, vol. 23, $\mathrm{n}^{\circ} 183,1980$.

TsAl, Accounting for dissolved gases in pump design, Chemical Engineering, July 26,1982 , vol. $89, \mathrm{n}^{\circ} 15$.

J. GÓLICH, Grandeurs caractéristiques de la similitude pour la capacité d'aspiration et l'extension des bulles dans les pompes, Revue technique Sulzer, 2/1980.

Adresse des auteurs:

Madame B. Le Fur

Messieurs J. F. David

D. Pécot

CETIM

74 , route de la Jonelière

44076 Nantes Cedex

Tél. : 1640373635
M. VERRY: Je voudrais faire un commentaire. Je crois qu'il y a une chose qu'il ne faut pas oublier de dire, c'est la manière dont on fait varier le NPSH dans la boucle fermée. Il est clair que si l'on a l'imprudence de faire varier le NPSH avec une vanne à l'aspiration de la boucle, ce que certaines personnes persistent à faire, les résultats que vous avez présentés ne sont plus du tout valables. En fait, l'air dissous se transforme en air libre dans la vanne et en aval, et on a un effet important comme on a pu le voir sur les courbes relatives à l'air libre.

Mme LE FUR: En ce qui concerne les essais que j’ai présentés sur l'influence de l'air occlus, j'ai dit qu'ils ont été faits par baisse du plan d'eau pour des raisons de qualité d'eau. Au début, on a essayé de les faire par vannage à l'aspiration mais on voyait aussitôt la cavitation de la vanne remonter vers la pompe. On avait un taux qui était loin d'être négligeable et de plus incalculable. On ne pouvait pas faire les essais dans ces conditions.

M. VERRY: Je suis tout à fait d'accord; les essais ont été tout à fait probants. Il faut en tirer la conclusion qu'il faut procéder comme vous le dites. Je voudrais faire une deuxième remarque qui est liée à la variation de l'influence du taux de gaz libre en fonction de la vitesse de rotation. On a l'impression, sur vos courbes, que lorsque la vitesse de rotation augmente, l'influence du taux de gaz libre devient beaucoup plus importante même en valeur relative. $\mathrm{Ne}$ pourrait-on pas penser que, si l'on est à faible vitesse de rotation, le gaz libre que l'on a injecté dans la tuyauterie d'aspiration subit une détente relativement faible lorsqu'il arrive depuis la tuyauterie d'aspiration jusqu'au col de la roue alors que, lorsqu'on a une grande vitesse de rotation, ce gaz libre subit une détente beaucoup plus importante ce qui conduit à un débit volumétrique de gaz plus important ? On pourrait ainsi expliquer cet effet. Je ne sais pas si l'on peut imaginer trouver un paramètre adimensionnel qui permette de tracer ces courbes de façon indépendante de la vitesse de rotation. Ce n'est peut-être pas facile parce qu'il est possible qu'interviennent simultanément plusieurs phénomènes de similitude différentes.

Mme LE FUR: Je ne l'ai pas essayé mais, en effet, ce serait une explication pour l'influence de la vitesse de rotation.

M. VERRY: Dernière remarque qui est liée au problème que vous avez eu pour arriver à garder un taux de gaz important lorsque vous faites baisser le NPSH. Je crois qu'il faut tirer la leçon du système de dégazage de M. LECOFFRE: lorsqu'on a des bulles dans la boucle, on dégaze et lorsqu'on a une vanne qui cavite, il y a des bulles. La solution pour arriver à baisser le NPSH sans dégazer est d'avoir une boucle qui ne cavite pas.

Mme LE FUR: Effectivement, dans le cas où la boucle fermée ne cavite pas, on ne dégazait pas.

Pour la pompe NS 20 le système de dégazage consistait en un hydro-éjecteur qui aspirait dans la bonbonne et on réinjectait cette eau dans la cheminée. Quand on était trop bas, on réinjectait de l'air pour augmenter le taux d'oxygène dissous. On n'a pas pu le faire pour la boucle NS 80 qui est une boucle beaucoup plus importante et qui n'avait pas le même système de dégazage.

M. MICHEL: Je voudrais poser deux questions. La première concerne la qualification des germes que vous injectez par votre système de grille. Ce système est décrit d'une manière globale et on ne sait pas très bien quelle est la taille des germes.

Mme LE FUR: On injecte de l'air à travers de tout petits trous de $2 / 10^{\mathrm{c}}$ de millimètre. On voit bien les bulles sortir de ces trous.

M. MICHEL : Deuxième question: Vous avez parlé de l'origine de la recherche. D'où vient le financement d'une telle recherche?

Mme LE FUR: Ce sont des recherches financées par la taxe parafiscale que les industries mécaniques versent au CETIM.

M. COURBIÈrE : Lorsque vous avez fait une corrélation qui n'est pas publiée dans le document entre les trois vitesses, j'ai noté que plus la vitesse était basse, plus vous étiez pessimiste dans les résultats. Ce n'est pas pour vous décourager car j'ai l'impression que d'autres hydrauliciens ont trouvé exactement la même chose ; cela signifie que, lorsqu'on a de grandes dimensions de pompes, on a intérêt à être prudent. Les lois de similitude sont très dangereuses.

Mme LE FUR: On a observé des différences pour les faibles vitesses, mais les corrélations ne sont pas mauvaises dans beaucoup de cas. C'est surtout pour les essais à 723 tours, pour une faible vitesse de rotation, que l'on a observé une forte divergence. 